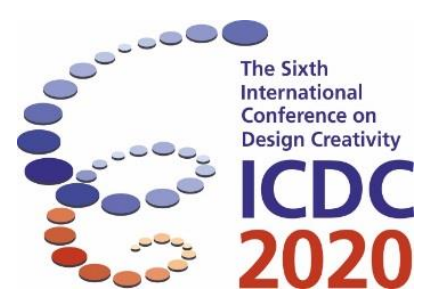

\title{
Design for One: Personalisation and experiences of design researchers and participants
}

\author{
Marjolein C. den Haan ${ }^{1}$, Rens Brankaert ${ }^{1,2}$ and Yuan $\mathrm{Lu}^{1}$ \\ ${ }^{1}$ Department Industrial Design, Eindhoven University of Technology, Eindhoven, The \\ Netherlands \\ ${ }^{2}$ Institute of Allied Health Professions, Fontys University of Applied Sciences, Eindhoven, \\ The Netherlands
}

\begin{abstract}
Personalisation in design is desired to address the uniqueness of people, and the design for one process is suitable to achieve this. However, more research is needed on people's experience with such a process to learn from it and improve it. We describe and examine the design for one processes of three different design cases, where young design researchers co-design with older adults to create personal designs. We found that the process is beneficial to interpret results better. However, the workload needs to be considered, and we can potentially extend further in personalizing the process by defining the users' creative strengths beforehand. Furthermore, we discuss applying this design for one process with other target groups and discuss the value of the unique findings of each particular case. Hereby, we contribute to how to execute and evaluate the design for one process and further enhance it for application in sensitive settings.
\end{abstract}

Keywords: design for one, co-design, personalisation

\section{Introduction}

In general, technology for ageing holds negative stereotypes and bias of old age that older adults experience in their everyday life (Lazar, Diaz, Brewer, Kim, \& Piper, 2017). Ageing has typically been framed for 30 years as a problem that has to be managed by technology (Vines, Pritchard, Wright, Olivier, \& Brittain, 2015). Leading to, for example, monitoring technology and care pendants, that adds little to enhance the lived experience of being older. This study aids in combatting that perspective by doing research through design for one, by bringing forward specific qualities of people. To avoid stereotypes, design researchers should look at people as individuals rather than representatives of a particular age group or disability (Pullin \& Newell, 2007). It implies that we need to act upon individual specifics to take their unique experiences into account. Older adults are a diverse group of people with different hobbies, interests, needs, and wishes (Cabrita et al., 2015; Gregor, Newell, \& Zajicek, 2002; Lerouge, Ma, Sneha, \& Tolle, 2011). To understand this variety, co-design with older adults has shown to be an effective way of including people's perspectives (Brereton, Soro, Vaisutis, \& Roe, 2015). With co-design people who are not trained in design creatively work together with designers in the design process (Sanders \& Stappers, 2008). We see the design for one process as a specific case illustrating co-design: "driven to suit the individual person (...) their preferences, abilities, and context." (Wilkinson \& Stones, 2018). One inspiring example we believe showcases the design for one approach is the case of Stephen Hawking. They gave Hawking a voice by custom made technology, but these 
developments also significantly contributed to text-to-speech software (Bertelsen, Bødker, Eriksson, Hoggan, \& Vermeulen, 2019). To better evaluate what contexts and disciplines benefit from the design for one processes, Wilkinson et al. (2018) suggest more research should be done around the designers' experiences executing these projects. Additionally, we want to incorporate the older adults' experience, because by being aware of both these experiences and reflect upon what works and doesn't work, we aim to improve the process (e.g., workload, challenges, and benefits) leading to better design. Therefore, we address the following research questions: What can we learn from the experiences of the design researchers and the older adults, to improve the design for one process? And how is personalisation expressed in the processes and designs?

\section{Related work}

\subsection{Co-design with older adults}

Processes in which older adults are involved to co-design include work on relatively far developed technologies such as mobile assistive technology (Hakobyan, Lumsden, \& O'Sullivan, 2015), media technologies (Botero \& Hyysalo, 2013), mobile devices (Massimi, Baecker, \& Wu, 2007) and software solutions (Sorgalla, Schabsky, Sachweh, Grates, \& Heite, 2017). Another example is the Un-Kit Experience where people can build personally meaningful IoT applications by a set of sensors, actuators, and media elements (Ambe et al., 2019). We start at the very beginning with involving older adults by searching for opportunities to let the design process evolve around the uniqueness of an individual.

\subsection{Personalisation towards one participant}

Focusing on one user specifically is not uncommon, as the four examples below show. The work of Orth and van den Hoven (2016) show that people's "unique identities and life narratives... reveal the potential for design to promote meaningful user-object relationships". Wallace et al. (2013) use their design probes to deeply engage with an older couple to design interventions to support their sense of self and relationships with others. Branco, Quental, and Ribeiro (2017) make artefacts personalised to make it more engaging to participate, based on ethics, empathy, and conviviality. Wilkinson and Stones (2018) challenged their students to design for a single person "to make this person 's life more pleasant". They are focusing on personal interaction, needs and abilities of a person rather than a user group. This related work shows the drive to create meaningful collaborations between a design researcher and a participant, as well as creating designs which are relevant and personal to the participant. We aim to investigate the design researchers' and participants' experiences of such a design process, and by reflecting on these, we aim to improve the process and our understanding of personalisation.

\section{Method}

We will describe how we set up the design process, the participants, and the data collection and analysis.

\subsection{Setting up the design process}

We asked three design researchers to conduct a design for one process with two older adults in parallel to personalise a design. Academic design research staff was involved in bi-weekly coaching of the design researchers. The project brief for the design researchers was to co-design "something" personal (e.g., product, service or system) with the participants. The process consisted of introductory session, discussing activities using the Leisure Time Canvas (den Haan, Brankaert, \& Lu, 2019), discussing ideas, and evaluating the prototype. All sessions were audio-recorded and transcribed anonymously by the design researchers.

\subsection{Involving our participants}

In table 1, we describe the three Industrial Design undergraduate students in their research semester and their participants. All older adults, who were recruited by partner organisations, signed a consent form 
after the project briefing. Thereby permitting audio recordings, taking unrecognisable pictures, and their awareness of being able to withdraw from the research at any point.

Table 1. Design researchers and participants information

\begin{tabular}{|c|c|c|}
\hline $\begin{array}{c}\text { Design researchers' age, gender } \\
\text { and project goal }\end{array}$ & $\begin{array}{c}\text { Participants' age } \\
\text { and gender }\end{array}$ & Participant characteristics \\
\hline $\begin{array}{c}\text { Naomi (22, F, Dutch) aims at } \\
\text { creating value for people with } \\
\text { memory challenges, by designing } \\
\text { to enrich their lives }\end{array}$ & Anna $(57, \mathrm{~F})$ & Enjoys music, diagnosed with Alzheimer's disease \\
\cline { 2 - 3 } & Bert $(88, \mathrm{M})$ & $\begin{array}{c}\text { Loves spending time with family and four lifelong } \\
\text { friends, diagnosed with early-stage dementia }\end{array}$ \\
\cline { 2 - 3 } $\begin{array}{c}\text { Gwen (21, F, Italian) aims at } \\
\text { exploring older adults' attitude } \\
\text { towards artificial intelligence by } \\
\text { designing open artefacts }\end{array}$ & Cor $(75, \mathrm{M})$ & Loves cooking and music, lives with a brain tumour \\
\cline { 2 - 3 } & Sophia $(75, \mathrm{~F})$ & Enjoys cycling, visiting museums and cooking \\
\hline $\begin{array}{c}\text { Vivian }(21, \mathrm{~F}, \text { Dutch) aims at } \\
\text { designing to empower and } \\
\text { socially engage older adults }\end{array}$ & Saar $(83, \mathrm{~F})$ & A caring lady who loves her voluntary work \\
\cline { 2 - 3 } & Johan (73, M) & A true storyteller and information gatherer \\
\cline { 2 - 3 } & Alice $(67, \mathrm{~F})$ & $\begin{array}{c}\text { A lady characterised by her sense of humour and } \\
\text { high ability to put things in perspective }\end{array}$ \\
\hline
\end{tabular}

\subsection{Collecting and analysing the data}

The second author conducted individual semi-structured interviews with the design researchers at the end of their project. The interviews were transcribed, and a thematic analysis was done following the six stages presented by Braun and Clarke (2006). Furthermore, we used their project reports to explain their processes and activities in section 4.

\section{Three cases}

This section shows a summary of the three cases with the personalised designs and the design processes.
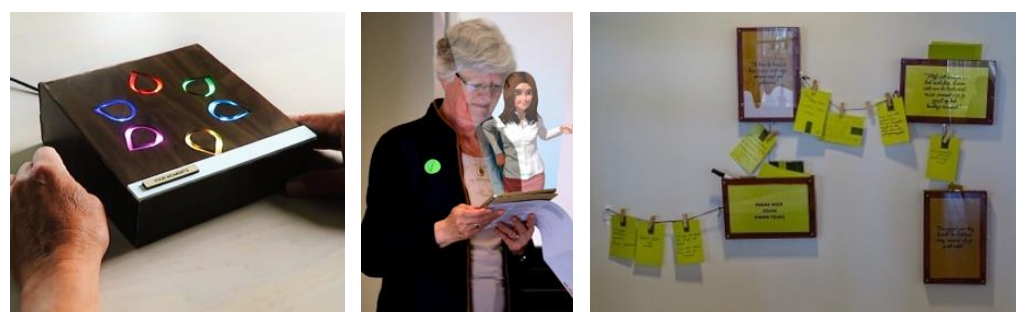

Figure 1. a) Case 1 Audio player, b) Case 2 Virtual assistants and

c) Case 3 Library of wisdoms (pictures made by the design researchers).

\subsection{The personalised designs of the three cases}

In case 1, Naomi created an audio player that could store personalised content, with music, audiobooks, and spoken memories - intended to listen to by themselves (see Figure 1a). This device for people with memory challenges stores personal memories of moments in their life to facilitate staying in touch with who you are.

In case 2, Gwen created virtual assistants to give recommendations for listening to music and visiting a museum (see Figure 1b). Through several co-creation sessions, Gwen facilitated her participants to formulate an opinion on what their virtual assistant should look like and let them experience how they perceive novel technology such as artificial intelligence.

In case 3, Vivian created a wall design to share wisdom quotes on paper tiles (see Figure 1c) because Saar used to give wisdom tiles to her friends as gifts. For Johan, something completely different, an audio player was created to store and playback audio fragments easily. The third involved participant, 
Alice, chose to collaborate in Saar's concept and develop it towards the library of wisdoms, where people could write and collect wisdom cards in photo frames.

\subsection{The design processes of the three cases}

In this study, design for one is based upon principles of co-design, yet we build the entire design process from beginning to end around one individual. We first get to know the person, secondly, create and test a personally tailored idea based on the person's background, experiences, and interests with him/her, then thirdly, create and evaluate a prototype to complete the design for one process. However, what we did differently than Wilkinson et al. (2018) is that our design researchers did two design for one processes in parallel because we wanted to stimulate reflection on unique participant characteristics and personalisation in the design.

So, the design researchers started two separate design processes with one individual, until a personal design was created for them (see Figure 2). We call these two design for one processes in parallel phase 1. After the first design outcome, they reflected on how to proceed: in case 1 one design is further developed with a third older adult, in case 2 the design is further developed with the same participants, and in case 3 participant Alice chose to continue with Saar's design. We call this phase 2, where the personal designs were developed further, in two cases with new participants and in one case with the same participants. By involving a new participant, we intended to reflect on to what extent the design was personalized and how the design would be different for a new person. The fact that the design researchers tuned their processes to address questions they found along the way shows already personalisation of the process.

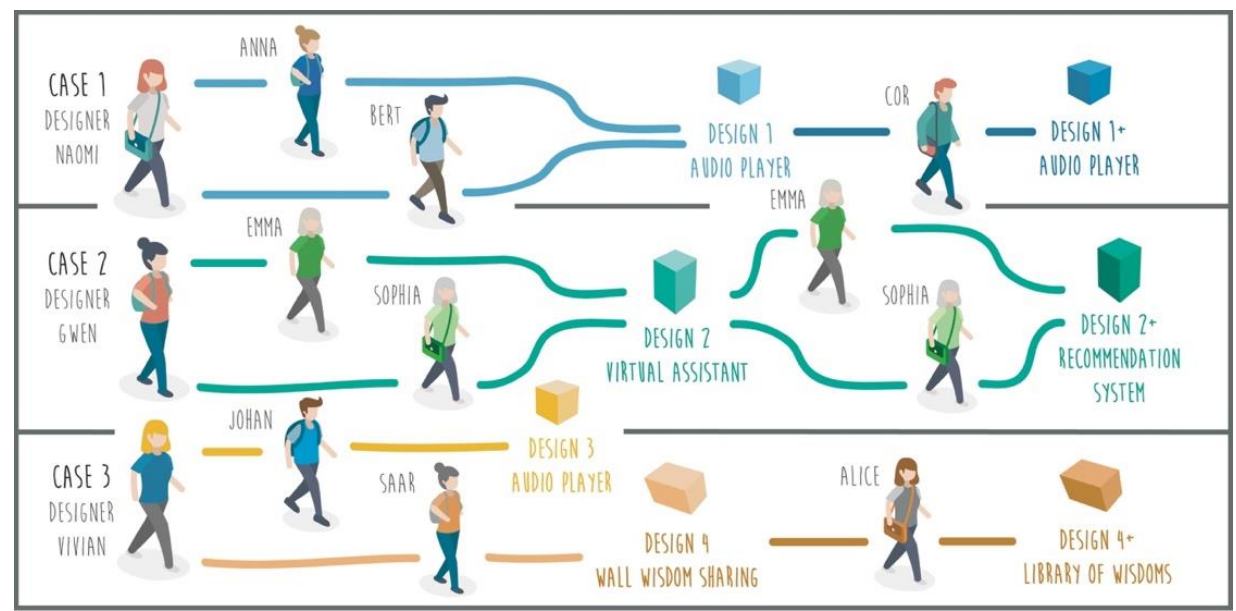

Figure 2. A visualisation of the design processes of the three cases.

\section{Result and analysis}

The interviews we conducted with every design researcher individually took place in June 2019 and lasted approximately 50 minutes. There were four categories of questions: 1) what they experienced as advantages and disadvantages of the project, 2) their learning moments, 3) personalisation in the design, and 4) personalisation in the process. We will describe these categories through the following two sections: the positive and negative design researchers' and participants' experiences per case, and secondly personalisation in the process and design.

\subsection{Case 1: Naomi aims at creating value for people with memory challenges by designing to enrich their lives}

Naomi found it valuable to get her participants' opinions quickly and frequently: "It's very beautiful, meaningful... it was very valuable to me to be able to iterate. ... and then go back to check what they think, back and forth.". She made her participants see what she's doing by creating an overview of her 
intended design process: "I tried to explain what it [the design process] included, but it was very difficult for them to understand without any image, a product. So, they found it difficult to think along with it. Bert found it a bit scary, but switch from reservedly to active participant.". At times though, Naomi had a hard time as a perfectionist: "Actually the plan that I prepared was fine, but then I start to worry." and "I have something in mind, and then I executed that, and then I think it would be nice to do this and this and this as well.".

\subsection{Case 2: Gwen aims at exploring older adults' attitude towards artificial intelligence by designing open artefacts}

Gwen found it challenging to start the project, as portrayed by the following quotes: "Yeah I really struggled because I had never done qualitative research before." and "You don't know what you need to find out and through this kind of study you can find the unknown unknown... and take it further. So that's what I think is so meaningful.". Though getting to know her participants helped her interpreting information: "And in this case, it was really nice to really get to know the person in front of you, so you're really able to analyse the results that you have in a personalised way. You have a clear idea of all the factors that are playing a role. Well, not all of them, but a lot of them. And then you have a much clearer idea on how to interpret the results or how to analyse the results that you have. (...) But if you know someone, because you have so much extra information on them, you understand what their ten is and what is their one.".

\subsection{Case 3: Vivian aims at designing to empower and socially engage older adults}

Vivian started the project a bit hesitant: "Wow, that sounds intense! [laughs] How am I going to do that? All those co-design sessions?". This feeling continued during her project as she found the projects' workload quite high. On the one hand, because of the time spent preparing the user sessions: "as designer, you have to prepare, organise and be in contact with your participants as well as the activity centre - all by yourself" and, on the other hand by being a perfectionist: "I have to do everything very elaborately". Then, to elaborate upon the participants' positive experiences: "Johan just really likes to talk to people, so he enjoyed being part of it. " Furthermore, empowerment was not just her goal, but it was also notable while executing her research: "Alice said she was really proud. Proud of the design. And that she was part of something that was actually present in the activity centre, and part of something which I could use for my research. And that she contributed to research." and "Saar felt empowered by the process, it gave her insights on her daily life, e.g., that she needed to accept help". However, Saar also considered dropping from the study: "Saar wanted to withdraw on a very important moment when I wanted to evaluate a concept with her. So, the activity centre arranged I could still do that with her.". Although Vivian did not want to force her participant to stay, Vivian was interested in understanding why Saar wanted to stop, so Vivian discussed this with a care professional in the activity centre who knew Saar very well. Thereby Vivian learned it was mostly a matter of insecurity, so Vivian should explain more clearly to Saar how much she valued Saar's contribution. This is interesting because we were aware that Vivian was learning a lot from Saar, and she also stated this herself: "Both very lovely people to have met and to learn from.", but apparently this did not land with Saar.

\subsection{Personalisation in the process and design}

In case 1, Naomi explains she still gave her participant priority over her wishes: "It's very personalised to the user, so I took that into account, and if I would make this [design] for myself, it would probably look very different.". Furthermore, in case 2 , Gwen facilitated her users to think along with a completely new topic for her users: "it evolved to a really interesting conversation [about artificial intelligence], they [her participants] really... they never talked about it before... and in industrial design, we talk about these topics while drinking a beer.". Vivian, from case 3, personalized the process to her participant Johan who was visually impaired: "I found ways to verbally explain my concepts [instead of showing sketches] or just only discuss the design areas for which the concepts were created.". And when the third participant, Alice, further designed Saar's personal design, we asked Saar whether the developed design still felt personal to her. She said: "Not at all actually." and Vivian adds: "She thought it looked nice, but it did not belong to her anymore.". 


\section{Discussion}

Throughout our study, we have gained insights on how to execute the design for one process.

Following our first research question, based on the experiences of the design researchers, we found ways to improve the design for one process. We found that visually communicating the timeline and contents of a session provides confidence to participate as a user. Furthermore, we found this more personal design process contributes to understanding the users and interpreting user sessions, as design researchers start to get to know the person. Though, we found that the design researchers sometimes struggled with the design for one process and suggest managing the workload better by doing the design for one process in teams and scope the data collection.

In addition, following our second research question, we found several expressions of personalization. We found personalization can be extended further in the process by defining the users' creative strengths beforehand. Furthermore, we discuss applying design for one process with other target groups. And we reflect on the success criteria of the project: we suggest to evaluate the impact of the design for one process beyond the design, and to what extent it affects people's daily life. To conclude, we discuss how to generalize the personal findings by cumulating the particular findings.

\subsection{Improving the design for one process based on the experiences of the design researchers and participants}

The following examples illustrate how we can improve the design for one process based on the experiences of the design researchers and participants.

Naomi's participants indicated uncertainty in the design process as they had difficulties in understanding her and the process aims without concrete visuals to respond to. Thereby the study suggests design researchers should clearly visualize the main aim or steps of a conversation, even it is an open session where we want to get inspired and are not searching to find specific answers. So, we should communicate clearly to participants that a session was fruitful.

Also, the design for one process enabled the design researcher to explore a for the participants unknown topic, as we have seen in Gwen's project, and make it concrete and formulate an opinion about it. In this way, participants can learn about something new and contribute to the design from their perspective. This can be beneficial to bring other new topics to participants as well.

Furthermore, Gwen explained that because she had so much extra information on them, she could better understand "what is their ten and what is their one" with regard to her participants assessing their experience with the prototype. In our study, the design researcher did both the fieldwork and the analysis, and this process would probably spark different results when executed by and shared with multiple design researchers. It may be a challenge to do this kind of personal process in teams, yet possible when one design researcher is in charge of the participant sessions, to build a relationship, and shares this knowledge with the rest of the team.

Secondly, the 'extra' information made the design researcher understand better how to interpret the results, yet we may have collected information that was less useful than others. One may argue if we know better what to collect beforehand, we may decrease the workload for both the participant and design researcher. Yet, we do value the little side-tracks in data gathering during design, as these may contribute later as well. Although it is a very time-consuming process to collaborate with one individual, it served the purpose of creating a personal design and was a way to include a participant who potentially would not share as much in a group setting: "Saar really enjoyed the way she was involved as it was less chaotic than in a group, which she could not handle that well.".

In addition, it remains important to involve professional caregivers to interpret the experiences of participants as the following example shows. Although Vivian really valued Saar's contribution, Saar felt insecure about her participation. The activity center who recruited the participants recommended to communicate more clearly about the value and expected input of the participants. This advice resulted in that Saar realized the project impacted her daily life practice positively.

Currently, the design for one process aims to deeply connect with a person and create something meaningful for this person. The end-stage is that the person tests the design outcome, to evaluate how the user experiences the personal prototype. However, the design for one process offers more. We saw in the case of Alice that she was proud of the design, proud to be part of the research, and proud it was 
actually presented in the activity center to her peers. The latter could be an interesting direction to include in the design for one process to involve recognition or acknowledgment by others, and using this process to achieve that.

\subsection{Personalisation in the processes and designs}

To address our second research question about personalization in processes and designs, we align with Hendriks, Slegers, \& Duysburgh (2015) who advocate for adjusting co-design techniques for people living with a cognitive or sensatory impairment towards a highly individual approach. This study shows the design researchers were flexible around the abilities of the participants. However, personalization could also be pushed further in defining the creative strengths of older adults before the design process starts. To let them (and facilitate them to) decide where they feel confident to be involved. This was shown in Vivian's process with Alice (design case 3), who continued on Saar's design. Though this case also showed that small changes in a design, while keeping the same idea, already highly influences to what extent the design is perceived as personal. All design cases showed how to adapt the activities and type of involvement more to the skills and interests of the participants, which is in line with (Wilkinson \& Stones, 2018) that methods cannot simply be taken from the shelf. One participant might be a true storyteller, while the other is more comfortable to reflect on ideas: "I think by already having something really concrete, it [a new person testing the previous design] was an easier transition.". Although Vivian describes a potential disadvantage: "I think because she [Saar] wasn't in the last phase anymore... it creates more distance.".

As Hendriks et al. (2015) also suggest, adapting co-design processes is not just for people with an impairment, but also for people with a 'situated disability' by which they mean that a person may have fully functional reading skills in a Western country, but becomes impaired when looking at travel signs in Arabic. People transitioning between life phases or going through traumatic events might experience something similar. So, we believe that this personal design for one process can be beneficial for many different people and target groups, learning about their interests and capabilities to design for.

All in all, it is challenging to assess when personalization is successful. Is it the level of excitement of the participant involved (process), or the potential of the design for a wider group as well? Perhaps one of the success criteria could be to determine its impact beyond the design. So, in the case of Saar (Vivian's participant), she learned, for example, that she needed to accept help in her daily life. This is something she realized in a session with the design researcher. Then about the potential of the design for a wider group, Vivian (design case 3) said: "You see you create a design which really fits a person, but on the other hand, shows so many opportunities to be translated to other people.". We were questioning to what extent a personal design can be useful for others. In the research of Bertelsen et al. (2019), they warn for generalization, the reality might become too abstract so that the results cannot be taken towards actual design questions. Instead, they argue for a cumulation of particulars, the specifics of each design case, which can be further examined through new design cases. If we look at our three cases through this lens, we would argue that from Naomi's design case 1, we take a general concept that can be personalized by any user in terms of content. From Gwen's design case 2, we see a process to let the user get acquainted with something unfamiliar by building something themselves. From Vivian's design case 3, you see the expression of certain values and views on life and how to share these with others in a community center. We can generally conclude that all designs show that a personal design process is a strong approach to making usable and acceptable ideas where users feel ownership of. Yet we also value the particular cases as stand-alone examples to show the fit of a design with a person in a particular context.

\subsection{The limitations}

We explored three cases, and although with a bigger sample size, we would potentially gain new insights, we believe it enabled us to gather insights of the design researchers and participant experiences. Also, because of coaching and guiding the design researchers by academic design research staff, we could have monitored the academic staffs' input to separate the design researchers' and academic staff's decisions in the project. 


\section{Conclusion}

We elaborated upon the design researchers and participants' experiences to reflect on the process and improve it so other design researchers can build further on this. The personalisation in the design for one process could be pushed even further by actively inviting older adults to activities they resonate with most and feel confident in. We have shown three cases that departed from the same starting point, and emerged into three very different cases, topics, and outcomes. By cumulating these particulars rather than generalise, we found how these meaningful personalised design process and outcomes contributed in their particular context.

\section{References}

Ambe, A. H., Brereton, M., Soro, A., Chai, M. Z., Buys, L., \& Roe, P. (2019). Older People Inventing their Personal Internet of Things with the IoT Un-Kit Experience, 1-15.

Bertelsen, O. W., Bødker, S., Eriksson, E., Hoggan, E., \& Vermeulen, J. (2019). Beyond Generalization: Research for the Very Particular. Interactions, 35-38.

Botero, A., \& Hyysalo, S. (2013). Ageing together: Steps towards evolutionary co-design in everyday practices. CoDesign, 9(1), 37-54.

Branco, R. M., Quental, J., \& Ribeiro, Ó. (2017). Personalised participation: an approach to involve people with dementia and their families in a participatory design project. CoDesign, 127-143.

Brereton, M., Soro, A., Vaisutis, K., \& Roe, P. (2015). The messaging kettle: Prototyping connection over a distance between adult children and older parents. In CHI '15 (pp. 713-716).

Cabrita, M., Nassabi, M. H., Akker, H. op den, Tabak, M., Hermens, H., \& Vollenbroek, M. (2015). An Unobtrusive System to Monitor Physical Functioning of the Older Adults: Results of a Pilot Study. In International Workshop on Personalization and Adaptation in Technology for Health held in conjunction with the 23rd Conference on User Modelling, Adaptation and Personalisation.

den Haan, M., Brankaert, R., \& Lu, Y. (2019). The Leisure Time Canvas: Eliciting Empathy for Older Adults through Activities and Hobbies. In Academy for Design Innovation Management.

Gregor, P., Newell, A., \& Zajicek, M. (2002). Designing for Dynamic Diversity - interfaces for older people. In Assets '02.

Hakobyan, L., Lumsden, J., \& O’Sullivan, D. (2015). How to Engage Older Adults in Participatory Design Activities. International Journal of Mobile Human Computer Interaction, 7(3), 78-92.

Hendriks, N., Slegers, K., \& Duysburgh, P. (2015). Codesign with people living with cognitive or sensory impairments: a case for method stories and uniqueness. CoDesign, 11(1), 70-82.

Lazar, A., Diaz, M., Brewer, R., Kim, C., \& Piper, A. M. (2017). Going Gray, Failure to Hire, and the Ick Factor: Analyzing How Older Bloggers Talk about Ageism. In CSCW' 17 (pp. 655-668).

Lerouge, C., Ma, J., Sneha, S., \& Tolle, K. (2011). User profiles and personas in the design and development of consumer health technologies. International Journal of Medical Informatics, 82(11), e251-e268.

Massimi, M., Baecker, R. M., \& Wu, M. (2007). Using participatory activities with seniors to critique, build, and evaluate mobile phones. In Assets '07 (pp. 155-162).

Orth, D., \& van den Hoven, E. (2016). "I wouldn't choose that key ring; it's not me": A Design Study of Cherished Possessions and the Self. In $\mathrm{OzCHI}$ ' 16 .

Pullin, G., \& Newell, A. (2007). Focussing on Extra-Ordinary Users. In UAHCI 2007 (pp. 253-262).

Sanders, \& Stappers, P. J. (2008). Co-creation and the new landscapes of design. CoDesign, 4(1), 5-18.

Sorgalla, J., Schabsky, P., Sachweh, S., Grates, M., \& Heite, E. (2017). Improving Representativeness in Participatory Design Processes with Elderly. In CHI EA '17.

Vines, J., Pritchard, G., Wright, P., Olivier, P., \& Brittain, K. (2015). An Age-Old Problem: Examining the Discourses of Ageing in HCI and Strategies for Future Research. ACM Transactions on Computer-Human Interaction, 22(1), 27.

Wallace, J., Wright, P. C., Mccarthy, J., Green, D. P., Thomas, J., \& Olivier, P. (2013). A Design-led Inquiry into Personhood in Dementia. In CHI '13 (pp. 2617-2626).

Wilkinson, A., \& Stones, C. (2018). Designing For One; How Adjusting Variables Influenced Design Student Creativity. In ICDC2018. 